output of West African cattle (and also of goats, sheep and poultry) at present than their inherent characteristics. Drought leads to shortage of feed and of water, and may lead to losses of 25 per cent of live weight in a single season. The improvement of water supplies by conservation requires planned use of grazing, while the problem of dry-season feed supplements has scarcely been tackled, partly because there is little information on the composition of animal feeds in the savannah regions. The West African ox does not seem to possess the superior digestive powers with which it is credited, and indeed digestibility is reduced when animals are fed on a low plane of nutrition, particularly in respect of phosphorus and protein. Basic work on all these matters, which would no doubt include studies of urea and other nonprotein nitrogen sources, is needed on the spot.

Introduced varieties of pigs and poultry can do well in West Africa. Pigmeat is not in demand, but great expansion of egg production is possible if the birds are properly fed and cared for and not regarded as mere scavengers. An industry based on imported chicks is developing, but serious nutritional problems (protein, vitamins $B$ and others, calcium and other minerals) remain to be overcome. An industry for feeding-stuffs could be based on local materials which are at present exported for the formulation of feeds in Europe.

The last speaker, Mr. R. S. Rack, based his contribution on his remarkable work in the practical development of native fishing industries in Northern Rhodesia. His address was notable for its active concern with the human problems of agricultural development. He pointed out that the people of undeveloped countries have their own technical and social adaptations to their environment, and that the technological basis of their economy cannot be altered in isolation from its personal and social context. This difficulty shows itself in attitudes to organization and work discipline (whether selfimposed or not) and to the employment of time, and in the obstacles often presented by traditional habits and customs. The simple poverty of the native fisherman, and his social and family obligations, may prohibit the accumulation of capital; the size of his undertaking may in any event be too small to allow a single technical step (such as the purchase of an outboard motor) unless his whole scale of operation can be altered to take full advantage of it. Other problems include the supply of fish, the availability of technically competent and equipped fishermen, and the existence or possibility of markets and suitable transport. These factors were illustrated by an account of the successful measures taken since 1951 to improve the supply of fish (whether fresh, as a staple food, or dried as a relish in a cereal diet) to the Rhodesian Copper Belt, which involved an ice plant, a boat-building school, a marketing advisory organization and the encouragement of African traders, and is to lead on to a fisheries school which will teach navigation, the handling of powered boats and book-keeping in addition to the techniques of catching and preserving fish. Another example arose from the development of a fishing industry among the traditionally non-fishing Tongas when they were resettled because of the construction of the Kariba Dam, and a third from the improvement of a sardinetype fishery on Lake Tanganyika.

In the discussion following the papers, an interesting food chain involving water hyacinth, pigs and fish was described by Prof. Stamp from Hong Kong, and some disagreement was evident on the usefulness of imported stock in the tropics.

The Food and Agriculture Organization report, already mentioned, also deals with human and organizational problems, on a regional scale, in two special chapters. One, on land reform and institutional change, surveys the situation in the main regions of the world and then discusses particular problems such as tenancy, financial support, marketing, settle. ment and community development. The second is on the organization of extension education and research in Africa, Asia and Latin Ameriea. Taken as a whole, the report is, as usual, a valuable and informative survey.

The meeting, and the report, justify a degree of optimism not only about the technical possibilities but also about the chances that they will be in part realized during the next two or three generations. The social and historical imponderables are too great to make any further prediction useful: but it is impossible to avoid the conclusion that a general limitation of human numbers is ultimately desirable if a measure of dignity and worthiness of human life is ever to be achieved.

A. H. Bunting

\title{
SCANNING TECHNIQUES IN BIOLOGICAL AND MEDICAL MICROSCOPY
}

\begin{abstract}
$\triangle$ CONFERENCE on the principles and applications of scanning methods in the microscopy of biological and medical specimens, arranged by the New York Academy of Sciences, was held in the Henry Hudson Hotel, New York, during October 5-6. Fourteen papers were presented in the four sessions of the meeting, and all but one were concerned with optical microscopy, the exception being on electron and X-ray scanning microscopy.

The main group of contributions ranged from the straight application of television in microscopical observation to the design of a complicated flyingspot system for sizing cells. M. J. Kopac (Graduate School of Arts and Sciences, New York University) described the use of closed-circuit television in
\end{abstract}

connexion with the micro-surgical technique he has developed for operating on single cells. At present the micro-instruments, of which there may be as many as eight in use in a single operation, are manipulated by hand. It is planned to adapt the electronic techniques used for tracking targets in the air so as to control the movement of microneedles and micropipettes with servo-motors.

Scanning techniques are especially useful for cytochemical studies of transient processes in cells. C. N. Loeser and S. S. West (School of Medicine, Western Reserve University, Cleveland, Ohio) have been using a highly sensitive 'Intensifier Image Orthicon' television camera to record fluorescence emission spectra from individual Ehrlich ascites cells, stained 
with acridine orange. The average uptake of dye per cell has been determined and correlated with the spectral measurements. For quantitative investigations in the ultra-violet, two approaches are being followed. G. Z. Williams (National Institutes of Health, Bethesda) and R. G. Neuhauser (Radio Corporation of America, Lancaster), described the use of a new 'Vidicon' television camera sensitive to the ultra-violet as an image storage detector and for time-lapse work. They obtain oscilloscope tracings of the changing ultra-violet absorption of single living cells, allowing deductions about reaction-rates. On the other hand, V. K. Zworykin and C. Berkeley (Rockefeller Institute, New York) have been exploring applications of the colour-translating television microscope. In this instrument, the specimen is illuminated by three selected ultra-violet wavelengths and the three images produced are electronically converted into images in three different colours on a television screen. It is being used for examining ultra-violet radiation damage, the streaming of protoplasm and the absorption of metabolites such as vitamin $A$.

A still wider range of application is open to the flying-spot technique, in which the specimen is explored point by point sequentially. The source of illumination is the flying spot on the screen of an oscilloscope, which is demagnified through the microscope to throw a moving probe on the specimen. The image is built up on a display tube, or the signal may be used directly for quantitative absorption measurements. With suitable phosphors any part of the spectrum can be used, or indeed soft X-rays. T. O. Caspersson (Karolinska Institutet, Stockholm) gave a masterly survey of the methods which can be used, and the factors influencing instrumental design, in the three main areas of investigation: analysis of single cells or cell structures with high precision, analysis at lower accuracy of large numbers of cells or cell structures, and investigations on living material, which are often only possible by semi-quantitative or even qualitative methods. He described a number of instruments in use, or in development, in his own laboratory for these different types of research, and discussed the choice between mechanical, optical and electronic systems of scanning.

Most of the remaining contributions were variations on these themes. J. G. Hoffman (University of Buffalo) described measurements of the nucleic acid content and the dimensions of mammalian cells and their nuclei, both by ultra-violet absorption in fresh cells and by absorption in the visible range in unfixed cells and in Feulgen-stained cells. $\mathrm{He}$ discussed the use of a digital computer to facilitate analysis of the absorption measurements as they are obtained. Britton Chance (Johnson Foundation for Medical Physics, Philadelphia) described investigations of respiratory enzymes by analysis of the fluorescence spectra excited in single cells. J. J. Freed and J. L. Engle (Institute for Cancer Research, Fox Chase, Philadelphia) discussed the advantage of using a vibrating mirror to produce the sean, since it allows monochromatic illumination at any desired wave-length. They described the instrument they have built for making repeated absorption measurements on single living cells. It was found that continuous exposure of ascites tumour cells for 30 min. at wave-lengths above $2400 \AA$. produced no observable radiation damage, whereas at $2400 \AA$., or below, the typical radiation damage syndrome was observed.
The chairman of the conference, P. O'B. Montgomery, who has been mainly responsible for pioneering flying-spot microscopy in the United States, described recent work in his laboratory (University of Texas Southwestern Medical School, Dallas). In addition to the development of a flying-spot interference microscope, he and his colleagues (W. A. Bonner and L. L. Hundley) have worked out techniques for simultaneous illumination of the specimen with both visible and ultra-violet light. In one version of the apparatus, the two images appear side by side on the display tube. Alternatively, a very small part of the specimen is imaged by ultra-violet while a larger field around it is imaged by visible light. In this way the progress of ultra-violet radiation damage on selected cells, or even on different parts of a single cell, can be followed.

One of the important uses of the flying-spot mieroscope is for counting and sizing particles, and instruments are commercially available for routine measurements of this type. Attempts are now being made to extend the technique to the 'recognition' and counting of specific types of cells in tissue sections or smears, by applying to microscopy some of the experience gained in the use of radar for target spotting. An instrument named the 'cytoanalyser' has been built for the mass screening of cervical and vaginal smears, to aid in the early diagnosis of cancer. It is best described as an analogue-digital computer connected with an electromechanical scanning system, and is the fruit of collaboration between the Sloan-Kettering Institute for Cancer Research, New York, the Ameri. can Cancer Society, the National Cancer Institute, and Airborne Instruments Laboratories of Deer Park, New York. Its general principles of construction were described by $W$. E. Tolles and $H$. P. Mansberg of the last-named, and its functions and preliminary results of its operation by Elaine Diacumakos and Emerson Day of the first-named institution. The biological situation is complex, and it seems to be too early to judge how successfully the instrument can replace the human eye in spotting aberrant cells, and so dispensing with the squads of observers now employed in checking smears under the microscope. In any event, these two papers were another striking illustration of the feeding back of experience from the field of war to the problems of peace.

The remaining contribution, by V. E. Cosslett (Cavendish Laboratory, Cambridge), described the extension of flying-spot methods to the X-ray end of the spectrum, with some reference to the related technique of scanning electron microscopy. Both have so far been mainly applied to metallurgical and mineralogical problems. Very recently A. D. G. Stewart (Engineering Laboratory, Cambridge) has built an ion gun into an electron scanning microscope, to produce etching of a specimen surface during continuous electron imaging. It seems likely to be useful in studying the structure of teeth and other mineralized tissues, as well as of metals and alloys. At the Cavendish Laboratory, an exploration is being made (with aid from the Medical Research Council) of the possible biological and medical applica. tions of the X-ray scanning microscope-more accurately described as a micromalyser. Some preliminary results were reported to the Conference, from the detection of foreign particles in lungs and other tissues to work on the mineralization of bone and teeth. An appreciable proportion of iron (up to 7 per cent) has been found in the surface enamel 
of the incisors of a number of rodents. The technique has obvious uses in identifying dust particles in atmospheric pollution and related problems. The recent extension of its scope to the lighter elements, beryllium and carbon, makes an investigation of the possibilities of organic analysis desirable. However, it is doubtful if it can be of value in cytochemistry until the spatial resolving power is improved appre- ciably beyond the present limit of $0.25 \mu$ and the detection limit to better than one part in ten thousand.

The conference was throughout stimulating, and provoked much lively discussion, which often led back to fundamentals. It was the first meeting of its kind, and very much justified the initiative of the organizers.
V. E. Cossletet

\section{TIME-RESOLVED SPECTROSCOPY}

\begin{abstract}
A CONFERENCE on "Spectroscopic Studies I using Time-Resolved Techniques" was held in Manchester on June 16, under the auspices of the Institute of Physics and the Physical Society.

A brief introductory address was given by Prof. H. S. Lipson, president of the Manchester Literary and Philosophical Society, who welcomed members of the conference to the new rooms of the Society. He pointed out that the meeting was the first to be held there by the Institute of Physics and the Physical Society since these new buildings were completed, and which indeed had only been opened in September 1960.
\end{abstract}

The joint secretaries of the conference were Dr. L. Bovey, Atomic Energy Research Establishment, Harwell, and Dr. A. H. Gabriel, Atomic Weapons Research Establishment, Aldermaston. On the programme seven communications were listed and an eighth was added by Dr. C. Breton, who described some recent French work on plasma. As in other recent international conferences the scope of timeresolved spectroscopic work on plasmas, or indeed on discharges in general, has been shown to have extended very widely since the end of the Second World War, at which time very little work in this field had been done. This new work has, of course, largely been prompted by the rapidly growing interest in high-temperature plasmas, partly because of the widespread interest in the possibility of obtaining thermonuclear reactions and, more recently, partly because of work on the so-called magnetohydrodynamic conversion of energy. In both these closely related fields spectroscopic techniques are pre-eminent. This is because such techniques do not normally interfere with the discharge being investigated and because of their great range and power. The need for time-resolved studies, often on a sub-microsecond scale, has also led to the development of advanced optical techniques, and the latter were very much in evidence at the present conference.

Although speakers from France, the United States and South Africa all made contributions, the majority were provided by various workers from the Atomic Energy Authority at Harwell and Aldermaston.

The first paper was given by Dr. A. H. Gabriel, who has been responsible at the Atomic Weapons Research Establishment for the development of much striking optical apparatus for plasma work. He spoke about a system incorporating a fast-rotating mirror camera and a medium quartz spectrograph, used, for example, in work on fast pinched discharges with electron densities varying in the region $10^{15}-10^{18}$ per c.c. and lasting a few $\mu$ sec. and time resolutions of the order of $0.1 \mu \mathrm{sec}$. or less. Dr. Gabriel then proceeded to describe various other optical techniques available, namely, an image-rotating device in which three mirrors are used with a vertical slit, and an apparatus for work in the vacuum ultra-violet region using near normal incidence and covering the wave-length range 2000-5000 $\AA$. In such an instrument it was pointed out that astigmatism of the concave grating presents a difficulty. The spectrum is recorded near the base of the normal on to the Rowland circle and since only a limited wave-length range can be covered on one setting the position of the entrance slit assem. bly is made movable. A resolution of 3- $\mathrm{mm}$. image displacement per $\mu$ sec. is obtained. In connexion with all this work a precision capillary light source for calibration in the visible and quartz ultra-violet has been developed which gives virtually uniform illumination for about $12 \mu$ sec. and with an effective black. body temperature of about $25,000^{\circ}$. Another source for vacuum ultra-violet work, based on that of W. R. S. Garton at the Imperial College of Science and Technology, was also described by Dr. Gabriel. Experiments at the Atomic Weapons Research Establishment that were mentioned include measurements of excitation temperatures, that is, state populations, etc., for various discharges, and studies of free-free continua in the temperature-range $10^{5}-2 \times 10^{8}$ degrees, and finally, Dr. Gabriel mentioned experiments involving observation of emission spectra of rapidly pinched discharges, for example, the influence of impurity lines at different times, and work on absorption spectroscopy using axial viewing from a hollow pinch in which electron densities could be obtained from a study of line widths.

The next speaker, Prof. J. D. Craggs of the University of Liverpool, described recent experiments on the measurement of line broadening due to the inhomogeneous field Stark effect in spark discharges (transient arcs). He briefly summarized the historical development of this technique, which is now widely used in plasma investigations, from the days when the statistical theory was considered adequate to the present time when a more accurate realization of the complexities of the effect, particularly with regard to the contribution made by electrons in the impact broadening process, has been reached. Recent experiments in Liverpool, notably by Dr. E. E. L. Mitchell, strongly support the recent theoretical work of Griem and Kolb in the United States. This is for the Balmer lines of hydrogen, but in view of the present importance of the impact broadening contribution of electrons, experiments have been carried out over the past two years in Liverpool by $\mathrm{Mr}$. Lim, who has simultaneously studied sodium and argon lines (notably the former) broadened by the impact effect and the statistical-impact broadening of the Balmer lines. These results again support the Griem-Kolb theory and direct attention to the possibility of making electron density measurements 